A. K. Layek*, Department of Mathematics, Bangabasi College of Commerce, 35, Scott Lane, Calcutta 700009, West Bengal, India

e-mail: bcc@cal.vsnl.net.in

\title{
GENERAL FORMULATIONS OF SOME THEOREMS OF CLUSTER SETS
}

\begin{abstract}
Two theorems on symmetry properties of cluster sets relative to a grill for the domain of the functions are proved here. One of these contains a result of Young [8] and its analogue for qualitative and other cluster sets. (Wilczynski [7] introduced the notion of qualitative cluster sets.) The other contains a result of Erdös and Piranian [2], one of Dolzhenko [1], and analogous results for other cluster sets.
\end{abstract}

\section{Introduction}

In this section we introduce basic notation. Throughout the paper $\mathbb{R}, \mathbb{E}_{2}$ and $\mathbb{H}$ are taken to represent the real line, complex plane, and the open upper half plane, respectively.

Definition 1. A collection $P$ of subsets of $\mathbb{R}$ (respectively $\mathbb{E}_{2}$ ) is called a grill $[6]$ in $\mathbb{R}$ (respectively $\mathbb{E}_{2}$ ) if

(i) $\emptyset \notin P$,

(ii) $A \in P$ and $A \subset B$ implies $B \in P$, and

(iii) $A \cup B \in P$ implies either $A \in P$ or $B \in P$.

If a collection $P$ satisfies (i), (ii) and

(iii) $^{\prime} \cup_{n=1}^{\infty} A_{n} \in P$ implies $A_{n} \in P$ for at least one $n$,

then $P$ is called a $\sigma$-grill. Clearly a $\sigma$-grill is a grill.

\footnotetext{
Key Words: grill, $\sigma$-grill, porous, $\sigma$-porous, countable, category, measure, Baire property.

Mathematical Reviews subject classification: 30D40

Received by the editors April 21, 1999

*The results in Section 3 are taken from the author's doctoral thesis written under the direction of S. N. Mukhopadhyay of Burdwan University.
} 
Definition 2. Let $P$ be a grill in $\mathbb{R}$ (respectively $\mathbb{E}_{2}$ ) and $E \subset \mathbb{R}$ (respectively $\mathbb{E}_{2}$ ). A point $y \in \mathbb{R}$ (respectively $\mathbb{E}_{2}$ ) is said to be a p-point of $E$ if $E \cap N_{r}(y) \in$ $P$ for all $r>0$, where $N_{r}(y)=(y-r, y) \cup(y, y+r)$ or $N_{r}(y)=\{z: z \in$ $\left.\mathbb{E}_{2},|z-y|<r\right\}$ according to the requirement. The set of all $p$-points of $E$ will be denoted by $E_{p}$.

It can be verified that the operator $E \rightarrow E \cup E_{p}$ is a Kuratowski-closure operator defined on the class of all subsets of $\mathbb{R}$ (respectively $\mathbb{E}_{2}$ ), and hence it will generate a topology for $\mathbb{R}$ (respectively $\mathbb{E}_{2}$ ).

For $\theta$ and $\varphi, 0<\theta<\varphi<\pi$, let

$$
S_{\theta \varphi}=\{z: z \in \mathbb{H}, \theta<\arg (z)<\varphi\} .
$$

Then $S_{\theta \varphi}=S$ is the sector in $\mathbb{H}$ with vertex at the origin. Let $S_{\theta \varphi}(x)=S(x)$ be the translate of $S$ and which is obtained by taking the origin at $x \in \mathbb{R}$. For $x \in \mathbb{R}$ and $r>0$, we also set

$$
K(x, r)=\{z: z \in \mathbb{H},|z-x|<r\} \text { and } S(x, r)=S(x) \cap K(x, r) .
$$

\section{The Single Variable Case}

In this section we shall consider the theorem for functions of a single variable.

Let $f: \mathbb{R} \rightarrow W$, where $W$ is a topological space. Let $P$ be a grill in $\mathbb{R}$. For $U \subset W$, set

$$
f^{-1}(U)=\{x: x \in \mathbb{R}, f(x) \cap U \neq \emptyset\} .
$$

Let $f$ be an one or multi-valued function. Then the right hand $P$-cluster set $C_{P}^{+}(f, x)$ of $f$ at $x \in \mathbb{R}$ is the set of all $w \in W$ such that for every open set $U$ of $W$ containing $w, f^{-1}(U) \cap(x, x+r) \in P$ for all $r>0$. The definition of $C_{P}^{-}(f, x)$, i.e. the left hand cluster set of $f$ at $x$, is similar and is obtained by replacing $(x, x+r)$ with $(x-r, x)$ from the definition of $C_{P}^{+}(f, x)$.

Now we shall prove the auxiliary lemmas and theorems.

Lemma 1. Let $P$ be a grill in $\mathbb{R}$ and $E \subset \mathbb{R}$ be arbitrary. Then the set $T$ of all points $x$ in $\mathbb{R}$ such that $(x, x+r) \cap E \in P$ for all $r>0$ but $(x-r, x) \cap E \notin P$ for some $r>0$ is countable.

Proof. For a positive integer $n$, let

$T_{n}(E)=\{x: x \in \mathbb{R}, E \cap(x, x+r) \in P$ for all $r>0$ and $E \cap(x-1 / n, x) \notin P\}$.

Then clearly,

$$
T \subset \cup_{n=1}^{\infty} T_{n}(E) .
$$


Suppose that for some $n=k, T_{k}(E)=T^{\prime}$ is uncountable. Let $x^{\prime} \in T^{\prime}$ be a two sided limit point of $T^{\prime}$. Let $\left\{x_{m}\right\} \subset T^{\prime}$ be a sequence converging to $x^{\prime}$ and $x_{m}<x_{m+1}<x^{\prime}$ for all $m$. Then there is $x_{p} \in\left\{x_{m}\right\}$ so that $x_{p} \in\left(x^{\prime}-1 / k, x^{\prime}\right)$. Since

$$
E \cap\left(x_{p}, x^{\prime}\right)=E \cap\left(x_{p}, x_{p}+\left(x^{\prime}-x_{p}\right)\right) \in P
$$

it follows that $E \cap\left(x^{\prime}-1 / k, x^{\prime}\right) \in P$, which contradicts the fact that $x^{\prime} \in T_{k}(E)$. Thus, each set $T_{n}(E)$ is countable and hence $T$ is a countable set.

Lemma 2. Let $P$ be a grill in $\mathbb{R}$ and $E \subset \mathbb{R}$ be arbitrary. Then the set $T^{\prime}$ of all points $x \in \mathbb{R}$, such that $E \cap(x, x+r) \notin P$ for some $r>0$ but $E \cap(x-r, x) \in P$ for all $r>0$, is countable.

The proof is similar to that of Lemma 1.

Theorem 1. Let $f: \mathbb{R} \rightarrow W$ be an one or multi-valued function, where $W$ is a second countable topological space, and let $P$ be a grill in $\mathbb{R}$. Then, except at most a countable set of points $x \in \mathbb{R}$,

$$
C_{P}^{+}(f, x)=C_{P}^{-}(f, x) .
$$

Proof. Let $L$ be the exceptional set of the theorem. Let $B=\left\{B_{n}\right\}$ be a countable basis for the topology of $W$ and let $f^{-1}\left(B_{n}\right)=E_{n}$ for $B_{n} \in B$. Let $x \in L$. Then $C_{P}^{+}(f, x) \neq C_{P}^{-}(f, x)$. If possible, let $w \in C_{P}^{+}(f, x) \backslash C_{P}^{-}(f, x)$. Then there is a $B_{m} \in B$ containing $w$ such that $E_{m} \cap(x, x+r) \in P$ for all $r>0$, but $E \cap(x-r, x) \notin P$ for some $r>0$. Hence $x \in T_{m}$, where $T_{m}$ is the set $T$ in Lemma 1 with $E=E_{m}$.

Again if there is a $w \in C_{P}^{-}(f, x) \backslash C_{P}^{+}(f, x)$, it can be shown that there is a positive integer $k$ such that $x \in T_{k}^{\prime}$, where $T_{k}^{\prime}$ is the set $T^{\prime}$ in Lemma 2 with $E=E_{k}$. Thus, it is proved that

$$
L \subset \cup\left(T_{m} \cup T_{k}^{\prime}\right),
$$

where the union is taken for all positive integers $m$ and $k$.

Since by Lemma 1 and Lemma 2 each $T_{m}$ and $T_{k}^{\prime}$ is countable, $L$ is a countable set, which completes the proof.

Now we discuss some consequences of Theorem 1.

(i) If $P$ is the collection of all non- void sets in $\mathbb{R}$ then clearly $P$ is a grill in $\mathbb{R}$ and the $P$-cluster sets are the ordinary cluster sets, and we get the following theorem of Young [8]. 
Example 1. If $f: \mathbb{R} \rightarrow \mathbb{R}$ is a one to one multi-valued function then, except for at most a countable set of points $x$ in $\mathbb{R}$,

$$
C^{+}(f, x)=C^{-}(f, x) .
$$

(ii) If $P$ is the collection of all second category sets in $\mathbb{R}$ then $P$ is also a grill in $\mathbb{R}$ and we get the following analogue of Young's theorem for qualitative cluster sets $C_{q}^{+}(f, x)$ and $C_{q}^{-}(f, x)$.

Example 2. If $f: \mathbb{R} \rightarrow \mathbb{R}$ is an one or multi-valued function then except for at most a countable set in $\mathbb{R}$,

$$
C_{q}^{+}(f, x)=C_{q}^{-}(f, x) .
$$

(iii) If $P$ is the collection of all sets of positive outer measure (in the Lebesgue sense) of $\mathbb{R}$ then $P$ is also a grill in $\mathbb{R}$. If the cluster sets relative to this grill are called quantitative cluster sets and are denoted by $C_{m}^{+}(f, x)$ and $C_{m}^{-}(f, x)$ (see [9], set $\left.M(f, x)\right)$, then we get a similar symmetry relation between these cluster sets too.

Similar results can also be obtained if we consider $P$ to be the collection of all uncountable sets of $\mathbb{R}$. Let the cluster sets relative to this grill be denoted by $C_{a}^{+}(f, x)$ and $C_{a}^{-}(f, x)$, and let them be called attributive cluster sets. Set $C_{a}(f, x)=C_{a}^{+}(f, x) \cup C_{a}^{-}(f, x)$. Now, we prove a result which will improve the result of Collingwood proved in the paper Cluster set theorems for arbitrary functions with applications to function theory, Ann. Acad. Sci. Fenn. Ser. AI. No. 336/8 (1963), 83-146.

Theorem 2. If $f: \mathbb{H} \rightarrow W$ is an one or multi-valued function, where $W$ is a compact and second countable topological space, then except at a countable set of points $x$ in $R$, every value of $f(x) \in C_{a}(f, x)$.

Proof. Let $B=\left\{B_{n}\right\}$ be a countable basis for the topology of $W$. Let

$$
E_{n}=f^{-1}\left(B_{n}\right)=\left\{x: x \in \mathbb{R}, f(x) \cap B_{n} \neq \emptyset\right\} .
$$

Let $K$ be the exceptional set of Theorem 2. Let $x \in K$. Then there is $w \in f(x)$ but $w \notin C_{a}(f, x)$. Since $w \notin C_{a}(f, x)$ so there are $B_{k} \in B$ containing $w$ and a positive integer $p$ such that $E_{k} \cap(x-1 / p, x+1 / p)$ is countable. Further, since $x \in E_{k}$ so $E_{k} \cap(x-1 / p, x+1 / p)=K_{k p}$ is a countable set containing $x$ and thus

$$
K \subset \cup_{n=1}^{\infty} \cup_{m=1}^{\infty} K_{n m} .
$$

Since each set $K_{n m}$ is countable so $K$ is a countable set, and the proof is complete. 
Remark. Since $C(f, x)$ contains the set $C_{a}(f, x)$ so Collingwood's result follows from Theorem 2.

Theorem $2^{\prime}$. If $f: \mathbb{R} \rightarrow W$ is an one or multi-valued function, where $W$ is a compact and second countable topological space, then except at a first category set of points $x$ in $\mathbb{R}$, every value of $f(x) \in C_{q}(f, x)$.

The proof is similar to that of Theorem 2 .

\section{Auxiliary Results}

To prove the auxiliary lemmas and the corresponding theorem we require the following definitions.

Definition 3. Let $P$ be a grill in $\mathbb{E}_{2}$. If $f: \mathbb{H} \rightarrow W$ is arbitrary, where $W$ is a topological space, then the $P$-cluster set $C_{P}(f, x)$ of $f$ at $x \in \mathbb{R}$ is the set of all $w$ in $W$ such that for every open set $U$ of $W$ containing $w$, $f^{-1}(U) \cap K(x, r) \in P$ for all $r>0$. Considering the sector $S(x, r)$ instead of $K(x, r)$ in the definition of $C_{P}(f, x)$, we get the definition of sectorial $P$ cluster set $C_{P}(f, x, S)$ of $f$ at $x$ in the sector $S$.

Definition 4. A set $F \subset \mathbb{R}$ is said to be porous at a point $x \in \mathbb{R}$ if

$$
\limsup _{r \rightarrow 0} \frac{\ell(x, r, F)}{r}>0,
$$

where $\ell(x, r, F)$ is the length of the largest open interval in the complement of $F$, which is entirely contained in $(x-r, x+r)$. A set $F$ is said to be porous if it is porous at all its points. A set is said to be $\sigma$-porous if it is a countable union of porous sets. It is clear that a $\sigma$-porous set is a first category set of measure zero, but Zajíček [10] constructed a perfect set of measure zero which is not a $\sigma$-porous set.

We shall prove the auxiliary lemmas and the the theorem in the sequel.

Lemma 3. Let $P$ be a $\sigma$-grill in $\mathbb{E}_{2}$. If $F \in P$ then there is at least one point $z \in F$ which is a p-point of $F$.

Proof. Suppose the contrary. Then for each point $z \in F$ there is a $r=r_{z}$ such that the neighborhood $N_{r}(z)$ of $z$ satisfies $F \cap N_{r}(z) \notin P$. Since $\mathbb{E}_{2}$ is second countable with respect to the usual topology for $\mathbb{E}_{2}$ and

$$
F=\cup\left\{F \cap N_{r}(z): z \in F\right\},
$$


so there is a countable set of points $z_{1}, z_{2}, \ldots$, such that

$$
F=\cup_{n=1}^{\infty}\left\{F \cap N_{r}\left(z_{n}\right): z_{n} \in F\right\} .
$$

Since $F \in P$ is a $\sigma$-grill, there is at least one member, say $F \cap N_{r}\left(z_{m}\right)$, such that $F \cap N_{r}\left(z_{m}\right) \in P$. This is a contradiction, thus the proof is complete.

Lemma 4. Let $P$ be a $\sigma$-grill in $\mathbb{E}_{2}$ and let $G \subset \mathbb{H}$ be arbitrary. Then the set $T$ of all points $x \in \mathbb{R}$ at which there are two sectors $S^{1}(x)$ and $S^{2}(x)$ such that $S^{1}(x, r) \cap G \in P$ for all $r>0$, but $S^{2}(x, r) \cap G \notin P$ for some $r>0$, is a $\sigma$-porous set.

Proof. If $S^{1} \subset S^{2}$ then $T$ is empty and the proof is complete. So we suppose that $S^{1} \not \subset S^{2}$. For rationals $i, j, k, l$ in $(0, \pi)$ with $[i, j] \cap[k, l]=\emptyset$, and a positive integer $n$, set

$$
T_{n i j k l}=\left\{x: x \in \mathbb{R}, S_{i j}(x, r) \cap G \in P \text { for all } r>0 \text {, and } S_{k l}(x, 1 / n) \cap G \notin P\right\} .
$$

Then it can be shown that

$$
T \subset \cup T_{n i j k l},
$$

where the union is taken for all positive integers $n$ and rationals $i, j, k, l$ in $(0, \pi)$ with $[i, j] \cap[k, l]=\emptyset$. If possible, let $T_{n i j k l}$ be non-porous. So by definition there is $x^{\prime} \in \hat{T}=T_{n i j k l}$ such that

$$
\lim _{r \rightarrow 0} \frac{\ell\left(x^{\prime}, r, \hat{T}\right)}{r}=0
$$

where $\ell\left(x^{\prime}, r, \hat{T}\right)$ is the length of the largest open interval in the complement of $\hat{T}$ and is entirely contained in $\left(x^{\prime}-r, x^{\prime}+r\right)$. Since $x^{\prime} \in \hat{T}, S_{i j}\left(x^{\prime}, r\right) \cap G \in P$ for all $r>0$. For definiteness, suppose that $0<i<j<k<l<\pi$, and set

$$
K=\frac{\sin (i) \sin (l-k)}{\sin (k) \sin (l-i)}
$$

Then from (2), for an arbitrary $\epsilon, 0<\epsilon<K / 2$, there exists $\eta>0$ such that

$$
\ell\left(x^{\prime}, r, T\right)<\epsilon \cdot r
$$

for all $r<\eta$. Since $\hat{T}$ is non-porous at $x^{\prime}$ therefore for all $x>x^{\prime},\left(x^{\prime}, x\right) \cap \hat{T} \neq \emptyset$. Let $y \in\left(x^{\prime}, x^{\prime}+\eta\right) \cap \hat{T}$ be such that $S_{i j}\left(x^{\prime}\right) \cap S_{k l}(y, 1 / n)$ is a quadrilateral. Since $G \cap S_{i j}\left(x^{\prime}, r\right) \in P$ for all $r>0$, so by Lemma 3 , there is $z_{0} \in S_{i j}\left(x^{\prime}, r\right) \cap G$ and a $x_{0} \in \mathbb{R}, x^{\prime}<x_{0}<y$ such that $z_{0}$ is a $p$-point of $G$ and $z_{0}$ lies on $L_{l}\left(x_{0}, 1 / n\right)$, where

$$
L_{\theta}(x, r)=\{z \in \mathbb{H}, \quad \arg (z-x)=\theta \text { and }|z-x|<r\}, \quad 0<\theta<\pi .
$$


Let $J_{x_{0}}$ be the open segment on $L_{i}\left(x^{\prime}\right)$ intercepted by $S_{k l}\left(x_{0}, 1 / n\right)$ and let $I_{x_{0}}$ be the open interval on $\mathbb{R}$ with the right end-point at $x_{0}$ and

$$
\left|I_{x_{0}}\right|=\left|J_{x_{0}}\right| \cdot \frac{\sin (k-i)}{\sin (k)},
$$

where $|\cdot|$ denotes the length. Then clearly

$$
\frac{\left|I_{x_{0}}\right|}{x_{0}-x^{\prime}}=K
$$

From (3),

$$
\ell\left(x^{\prime}, x_{0}-x^{\prime}, \hat{T}\right)<\epsilon \cdot\left(x_{0}-x^{\prime}\right)
$$

and $0<\epsilon<K / 2$, therefore (4) ensures that there is a point $x^{\prime \prime} \in I_{x_{0}} \cap \hat{T}$ such that $z_{0} \in S_{k l}\left(x^{\prime \prime}, 1 / n\right)$. Since $z_{0}$ is a $p$-point of $G$, this implies that $S_{k l}\left(x^{\prime \prime}, 1 / n\right) \cap G \in P$, which contradicts the fact that $x^{\prime \prime} \in \hat{T}=T_{n i j k l}$. If we suppose that $0<k<l<i<j<\pi$ then we can arrive at a contradiction by proceeding from the left of $x^{\prime}$. Thus each set $T_{n i j k l}$ is porous, and the proof is complete by (1).

Lemma 5. Let $P$ be a $\sigma$-grill in $\mathbb{E}_{2}$ and $F \subset \mathbb{H}$ be arbitrary. Then the set $K$ of all points $x \in \mathbb{R}$ at which there is a sector $S(x)$ so that $K(x, r) \cap F \in P$ for all $r>0$, but $S(x, r) \cap F \notin P$ for some $r>0$, is a first category set in $\mathbb{R}$.

Proof. For fixed positive integer $n$ and rationals $i, j$ in $(0, \pi)$ with $i<j$, let

$$
K_{n i j}=\left\{x: x \in \mathbb{R}, K(x, r) \cap F \in P \text { for all } r>0 \text {, but } S_{i j}(x, 1 / n) \cap F \notin P\right\} .
$$

Then clearly $K \subset \cup K_{n i j}$, where the union is taken for all positive integers $n$ and rationals $i, j$ in $(0, \pi)$ with $i<j$.

If possible, suppose that $K_{n i j}=K^{\prime}$ is dense in an open interval $I\left(x^{\prime}\right)$, where $x^{\prime} \in K^{\prime}$ is the center of $I\left(x^{\prime}\right)$. Since for $x \in K^{\prime}, S_{i j}(x, 1 / n) \cap F \notin P$ and $K_{n i j}$ is dense in $I\left(x^{\prime}\right)$, so by Lemma $3, S_{i j}(x, 1 / n) \cap F \notin P$ for all $x \in I\left(x^{\prime}\right)$. Let

$$
B=\cup\left\{S_{i j}(x, 1 / n): x \in I\left(x^{\prime}\right)\right\} .
$$

Then we can choose a $r^{\prime}>0$ such that $K\left(x^{\prime}, r^{\prime}\right) \subset B$. Since for $x \in I\left(x^{\prime}\right)$, $S_{i j}(x, 1 / n) \cap F \notin P$, so by Lemma $3, B \cap F \notin P$, and hence $K\left(x^{\prime}, r^{\prime}\right) \cap F \notin P$. This contradicts the fact that $x^{\prime} \in K^{\prime}=K_{n i j}$. Thus, each set $K_{n i j}$ is nowhere dense in $\mathbb{R}$, so by (1) the set $K$ is a first category set. This completes the proof.

Theorem 3. Let $P$ be a $\sigma$-grill in $\mathbb{E}_{2}$ and let $f: \mathbb{H} \rightarrow W$ be arbitrary, where $W$ is a second countable topological space. Then 
(i) except at a first category set of points $x \in \mathbb{R}$, for each sector $S$ in $\mathbb{H}$

$$
C_{P}(f, x)=C_{P}(f, x, S) \quad \text { and }
$$

(ii) except at a $\sigma$-porous set of points $x \in \mathbb{R}$, for each pair of sectors $S^{1}$ and $S^{2}$ in $\mathbb{H}$

$$
C_{P}\left(f, x, S^{1}\right)=C_{P}\left(f, x, S^{2}\right) .
$$

Proof. Let $B=\left\{B_{n}\right\}$ be a countable basis for the topology of $W$ and set $f^{-1}\left(B_{n}\right)=E_{n}$ for $B_{n} \in B$.

(i) Let $L$ be the exceptional set of the first part of the theorem. If $x \in L$ then there is a sector $S$ in $\mathbb{H}$ such that $C_{P}(f, x) \not \subset C_{P}(f, x, S)$. Let $w \in$ $C_{P}(f, x) \backslash C_{P}(f, x, S)$. Then there is $B_{m} \in B$ containing $w$ such that $K(x, r) \cap$ $E_{m} \in P$ for all $r>0$, but $S(x, r) \cap E_{m} \notin P$ for some $r>0$. Thus, it is proved that $x \in K_{m}$, where $K_{m}$ is the set $K$ in Lemma 5 with $F=E_{m}$, and hence we get

$$
L \subset \cup_{n=1}^{\infty} K_{n} .
$$

By Lemma 5, each set $K_{n}$ is a first category set and therefore $L$ is a first category set. This completes the proof of the first part.

(ii) Let $L^{\prime}$ be the exceptional set of the second part of the theorem. Let $x \in L^{\prime}$. Then there is a pair of sectors $S_{1}$ and $S_{2}$ in $\mathbb{H}$ such that $C_{P}\left(f, x, S_{1}\right) \neq$ $C_{P}\left(f, x, S_{2}\right)$. Let $w \in C_{P}\left(f, x, S_{1}\right) \triangle C_{P}\left(f, x, S_{2}\right)$. Then there is $B_{k} \in B$ containing $w$ such that either

$S_{1}(x, r) \cap E_{k} \in P$ for all $r>0$ and $S_{2}(x, r) \cap E_{k} \notin P$ for some $r>0$, or

$S_{1}(x, r) \cap E_{k} \notin P$ for some $r>0$ and $S_{2}(x, r) \cap E_{k} \in P$ for all $r>0$, Hence in either case $x \in T_{k}$, where $T_{k}$ is the set $T$ in Lemma 4 with $E_{k}=G$, and so we have proved that

$$
L^{\prime} \subset \cup_{n=1}^{\infty} T_{n}
$$

By Lemma 4 , each set $T_{n}$ is a $\sigma$-porous set and therefore $L^{\prime}$ is a $\sigma$-porous set. This completes the proof of the theorem.

The above theorem includes several known results of ordinary cluster sets and it also generates the corresponding analogue for qualitative cluster sets. For example, let $P$ be the collection of all non-void subsets of $\mathbb{E}_{2}$. Then $P$ is a $\sigma$-grill and the $P$-cluster sets are the ordinary cluster sets $C(f, x)$ and $C(f, x, S)$. Applying Theorem 3, we get the following results. 
Example 3. ([2]). If $f: \mathbb{H} \rightarrow W$ is arbitrary, where $W$ is a second countable topological space, then except for a first category set of points $x$ in $\mathbb{R}$,

$$
C(f, x)=C(f, x, S)
$$

for each sector $S$ in $\mathbb{H}$.

Example 4. ([1]). If $f: \mathbb{H} \rightarrow W$ is arbitrary, where $W$ is a second countable topological space, then except for a $\sigma$-porous set of points $x$ in $\mathbb{R}$,

$$
C\left(f, x, S_{1}\right)=C\left(f, x, S_{2}\right)
$$

for each pair of sectors $S_{1}$ and $S_{2}$ in $H$.

If $P$ is the collection of all second category subsets of $\mathbb{E}_{2}$, then $P$ is also a $\sigma$-grill in $\mathbb{E}_{2}$ and the $P$-cluster sets are qualitative cluster sets $C_{q}(f, x)$ and $C_{q}(f, x, S)$. We get the following results from Theorem 3 .

Example 5. If $f: \mathbb{H} \rightarrow W$ is arbitrary, where $W$ is a second countable topological space, then except for a first category set of points $x$ in $\mathbb{R}$,

$$
C_{q}(f, x)=C_{q}(f, x, S)
$$

for every sector $S$ in $H$.

Example 6. If $f: \mathbb{H} \rightarrow W$ is arbitrary, where $W$ is a second countable topological space, then except for a $\sigma$-porous set of points $x$ in $\mathbb{R}$,

$$
C_{q}\left(f, x, S_{1}\right)=C_{q}\left(f, x, S_{2}\right)
$$

for each pair of sectors $S_{1}$ and $S_{2}$ in $H$.

Many other results can also be deduced from Theorem 3. For taking $P$ to be the $\sigma$-grill of all subsets of positive Lebesgue outer measure in $\mathbb{E}_{2}, C_{P}(f, x)$ and $C_{P}(f, x, S)$ become the quantitative cluster sets $C_{m}(f, x)$ and $C_{m}(f, x, S)$ [9]. We can deduce analogous results relating to these cluster sets too.

\section{The Main Results}

Here we shall prove a result which together with the results in the above examples will imply the results in [3] and [4].

For $\theta \in(0, \pi)$ and $x \in \mathbb{R}$, set

$$
L_{\theta}(x)=\{z: z \in \mathbb{H}, \arg (z-x)=\theta\}
$$


and

$$
L_{\theta}(x, r)=\left\{z: z \in L_{\theta}(x),|z-x|<r\right\} .
$$

In the sequel, for convenience, we have often written f.c. and s.c. for the terms first category and second category respectively. We have taken $W$ to be a second countable topological space whenever nothing is mentioned about $W$. Whenever other restrictions are needed for $W$, only those additional restrictions are mentioned.

Now we recollect some definitions which will be used in the lemma and the corresponding theorem.

Definition 5. A set $K \subset \mathbb{E}_{2}$ is said to have the Baire property if $K=$ $G \triangle Q$, where $G$ is an open set and $Q$ is a first category set in $\mathbb{E}_{2}$. A function $f: \mathbb{H} \rightarrow W$ is said to have the Baire property if for every open set $V$ in $W$, $f^{-1}(V)$ has the Baire property.

Definition 6. Let $f: \mathbb{H} \rightarrow W$. The directional cluster set $C(f, x, \theta)$ of $f$ at $x \in \mathbb{R}$ and in the direction $\theta \in(0, \pi)$ is the set of all $w \in W$ such that for every open set $U$ of $W$ containing $w, f^{-1}(U) \cap L_{\theta}(x, r) \neq \emptyset$ for all $r>0$. The definition of the directional qualitative cluster set $C_{q}(f, x, \theta)$ is the same as that of $C(f, x, \theta)$ but the condition " $f^{-1}(U) \cap L_{\theta}(x, r) \neq \emptyset$ " is to be replaced by " $f^{-1}(U) \cap L_{\theta}(x, r)$ is a s.c. set".

In the sequel $\{S\}$ will denote the collection of all sectors $S$ in $\mathbb{H}$.

Lemma 6. If $E \subset \mathbb{H}$ has the Baire property then at each $x \in \mathbb{R}$ the set

$$
\begin{aligned}
\Theta(E, x)= & \left\{\theta: 0<\theta<\pi, E \cap L_{\theta}(x, r) \text { is a f.c. set in } L_{\theta}(x) \text { for some } r>0,\right. \\
& \text { but } E \cap S(x, r) \text { is a s.c. set for all } r>0, \text { and each } S \in\{S\}\}
\end{aligned}
$$

is a f.c. set in $(0, \pi)$.

Proof. Let $E=G \triangle Q$, where $G$ is an open set and $Q$ is a f.c. set in $\mathbb{H}$. For a positive integer $n$, set

$$
\begin{aligned}
\Theta_{n}(E, x)= & \left\{\theta: 0<\theta<\pi, E \cap L_{\theta}(x, 1 / n)\right. \text { is a f.c. set, } \\
& \text { but for each } S \in\{S\}, G \cap S(x, r) \text { is a s.c. set for all } r>0\} .
\end{aligned}
$$

Then clearly $\Theta(E, x) \subset \cup_{n=1}^{\infty} \Theta_{n}(E, x)$. Suppose that $\Theta_{n}(E, x)$ is a second category set in $(0, \pi)$. Let

$$
\mathcal{V}(Q, x)=\left\{\theta: 0<\theta<\pi, L_{\theta}(x, r) \cap Q \text { is a s.c. set in } L_{\theta}(x) \text { for } r>0\right\} .
$$


Then by the Kuratowski-Ulam Theorem [5, p. 56] the set $\mathcal{V}(Q, x)$ is of first category in $(0, \pi)$. Thus $\Theta=\Theta_{n}(E, x) \backslash \mathcal{V}(Q, x)$ is a s.c. set in $(0, \pi)$. Therefore for each $\theta \in \Theta$ each set $L_{\theta}(x, 1 / n) \cap Q$ and $L_{\theta}(x, 1 / n) \cap E$ is of first category in $L_{\theta}(x)$. Thus, each set $L_{\theta}(x, 1 / n) \cap(G \backslash Q)$ and $L_{\theta}(x, 1 / n) \cap(G \cap Q)$ is a $f . c$. set in $L_{\theta}(x)$ for $\theta \in \Theta$. This implies that $L_{\theta}(x, 1 / n) \cap G=\emptyset$ for $\theta \in \Theta$. Since $\Theta$ is a s.c. set, we can suppose that $\Theta$ is dense in some interval $(i, j) \subset(0, \pi)$. The facts that $G$ is open and $\Theta$ is dense in $(i, j)$ ensure that $L_{\theta}(x, 1 / n) \cap G=\emptyset$ for $\theta \in(i, j)$. Thus, we get $S_{i j}(x, 1 / n) \cap G=\emptyset$. This is a contradiction because $S_{i j} \in\{S\}$. This proves that each set $\Theta_{n}(E, x)$ is a $f . c$. set in $(0, \pi)$, and hence $\Theta(E, x)$ is a f.c. set in $(0, \pi)$, which completes the proof.

Theorem 4. If $f: \mathbb{H} \rightarrow W$ has the Baire property then at each $x \in \mathbb{R}$ the set $\Theta(x)=\left\{\theta: 0<\theta<\pi, \cap_{S \in\{S\}} C_{q}(f, x, S) \subset C_{q}(f, x, \theta)\right\}$ is residual in $(0, \pi)$.

Proof. Let $B=\left\{B_{n}\right\}$ be a countable basis for the topology of $W$. Set $E_{n}=f^{-1}\left(B_{n}\right)$ for $B_{n} \in B$. Let $\theta \in(0, \pi) \backslash \Theta(x)$. Then there is a $w \in$ $\cap_{S \in\{S\}} C_{q}(f, x, S) \backslash C_{q}(f, x, \theta)$. So there is a $B_{m} \in B$ containing $w$ such that $E_{m} \cap S(x, r)$ is a s.c. set for all $r>0$ and each $S \in\{S\}$, but $E_{m} \cap L_{\theta}(x, r)$ is a f.c. set for some $r>0$. These prove that $\theta \in \Theta\left(E_{m}, x\right)$, where $\Theta\left(E_{m}, x\right)$ is the set $\Theta(E, x)$ of Lemma 6 with $E=E_{m}$. Thus, it is proved that

$$
(0, \pi) \backslash \Theta(x) \subset \cup_{n=1}^{\infty} \Theta\left(E_{n}, x\right) .
$$

By Lemma 6, each set $\Theta\left(E_{n}, x\right)$ is a f.c. set, hence $\Theta(x)$ is residual in $(0, \pi)$, and the proof is complete.

Corollary 1. Let $f: \mathbb{H} \rightarrow W$ have the Baire property. Then, except for an at most first category set of points $x$ in $\mathbb{R}$, the set $\mathcal{V}(x)=\{\theta: 0<\theta<$ $\left.\pi, C_{q}(f, x) \subset C_{q}(f, x, \theta)\right\}$ is residual in $(0, \pi)$.

Proof. The proof follows from the results in Theorem 4 and Example 5.

Corollary 2. Let $f: \mathbb{H} \rightarrow W$ have the Baire property. Then, except for at most a $\sigma$-porous set of points in $\mathbb{R}$, the set

$$
\Theta(x)=\left\{\theta: 0<\theta<\pi, \cup_{S \in\{S\}} C_{q}(f, x, S) \subset C_{q}(f, x, \theta)\right\}
$$

is residual in $(0, \pi)$.

Proof. The proof follows from the results in Theorem 4 and Example 6 .

Corollary 3. Let $f: \mathbb{H} \rightarrow W$ have the Baire property, where $W$ is also compact. Then, except for at most a $\sigma$-porous set of points $x$ in $\mathbb{R}$, there exists a residual set $\Phi(x)$ in $(0, \pi)$ at $x \in \mathbb{R}$ such that $\cap_{\theta \in \Phi(x)} C(f, x, \theta) \neq \emptyset$. 
Proof. The proof follows from the result of Corollary 2 together with the fact that $C_{q}(f, x, \theta) \subset C(f, x, \theta)$, and $C_{q}(f, x, S) \neq \emptyset$ for a compact $W$.

\section{Remarks.}

(i) The set inequalities contained in the relations in Corollary 1 and in Corollary 2 can be strengthened to equality if we use the fact that for rationals $i<j$ in $(0, \pi), C_{q}(f, x, \theta) \subset \cup_{0<i<j<\pi} C_{q}\left(f, x, S_{i j}\right)$ for a residual set of directions $\theta$ in $(0, \pi)$.

(ii) Since for a continuous $f, C_{q}(f, x, \theta)=C(f, x, \theta), C_{q}(f, x)=C(f, x)$ and $C_{q}(f, x, S)=C(f, x, S)$, the results in Corollary 1 and in Corollary 2 become results for ordinary cluster sets in this case.

\section{References}

[1] E. P. Dolzhenko, Boundary properties of arbitrary functions, Izv. Acad. Nauk SSSR, Ser. Mat. (1967).

[2] P. Erdös and G. Piranian, Restricted cluster sets, Math. Nach. 22(1960), $155-158$.

[3] M. J. Evans and P. D. Humke, On qualitative cluster sets, Colloq. Math. 37(1977), 255-261.

[4] A. K. Layek, On sectorial qualitative cluster sets and directional qualitative cluster sets, Colloq. Math. 50(1986), 281-288.

[5] J. C. Oxtoby, Measure and category, Springer-Verlag, New York Heidelberg Berlin, 1971.

[6] W. J. Thron, Proximity structures and grill, Math. Ann. 206(1973), 3562.

[7] W. Wilczynski, Qualitative cluster sets, Colloq. Math. 32(1974), 113-116.

[8] W. H. Young, On some applications of semi-continuous functions, Atti del IV Congresso Internationals dei Matematici (Roma 1908) II Roma (1909), 49-60.

[9] L. Zajíček, On cluster sets of arbitrary functions, Fund. Math. 83(1974), 197-217.

[10] L. Zajíček, Sets of $\sigma$-porosity and sets of $\sigma$-porosity(q), Casopis Pest. Mat. 101(1976), 350-359. 